\title{
Report from European Association for the Study of the Liver: HCC Summit, Geneva, Switzerland, 2-5 February 2017
}

\author{
Rodolfo Sacco ${ }^{*, 1}$, Aurora Mirabile², Luca Giacomelli³, Giampaolo Bresci', \\ Simona Attardo ${ }^{4} \&$ Giuseppe Cabibbo 4 \\ The European Association for the Study of the Liver Hepatocellular Carcinoma (HCC) \\ international meeting, Geneva, Switzerland, 2-5 February 2017
}

The European Association for the Study of the Liver Hepatocellular Carcinoma (HCC) international meeting held in Geneva in February 2017 focused on the state of the art of HCC management, from diagnosis to treatment and the potential development of clinical research in this field. This report reviews some of the most interesting topics discussed at the meeting such as the role of hepatitis $C$ viral infection treatment with direct-acting antivirals in enhancing HCC risk, current prognostic systems, early diagnosis techniques, curative therapies for early HCC and the systemic treatments for advanced disease with a look into future perspectives.

First draft submitted: 20 March 2017; Accepted for publication: 20 April 2017; Published online: 7 June 2017

Hepatocellular carcinoma (HCC) is a major health problem, with increasing incidence in Western countries, and it is the third highest cause of cancer-related death globally, following lung and stomach cancers [1,2]. In the west, the disease is diagnosed in $30-40 \%$ of all patients at early stages and is amenable to potentially curative treatments, such as surgical therapies (resection and liver transplantation) and thermal ablation [3]. At intermediate stage, patients can be treated with transarterial chemoembolization (TACE). However, disease diagnosed at advanced stage or with progression after locoregional therapy has a dismal prognosis, owing to the underlying liver disease and lack of effective treatment options [3,4]. In 2017 conference, held on 2-5 February in Geneva, the most recent progresses in etiology, diagnostic and staging procedures, management and treatment of liver cancer have been presented.

\section{Etiology, diagnostic \& staging procedures}

The risk of HCC depends on a background of chronic liver disease, including chronic hepatitis C (HCV) or hepatitis B viral infection (2-5\%), genetic predisposition, older age and abuse of alcohol [5].

In relation to the availability of modern antiviral therapies, it is foreseen that in the future the etiology of cirrhosis may likely appear more related to metabolic forms than to hepatotropic virus' infections.

Nevertheless, the role of HCV therapy in enhancing HCC risk has been largely debated in the last year. In fact, in 2016 Reig et al. showed an unexpected high rate and pattern of tumor

'Section of Gastroenterology \& Metabolic Disorders, Cisanello Hospital, Pisa, Italy

2Department of Oncology, Division of Experimental Oncology, IRCCS San Raffaele Scientific Institute, Milan, Italy

${ }^{3}$ Department of Surgical Sciences \& Integrated Diagnostics, School of Medicine, University of Genoa, Genoa, Italy

${ }^{4}$ Section of Gastroenterology, Biomedical Department of Internal \& Specialized Medicine (DIBIMIS), University of Palermo, Palermo,

Italy

*Author for correspondence: r.sacco@ao-pisa.toscana.it

\section{KEYWORDS}

- EASL summit • HCC • HCC diagnosis $\bullet \mathrm{HCC}$ treatment 
recurrence coinciding with $\mathrm{HCV}$ clearance after direct-acting antiviral (DAA) treatment in patients with prior HCC assuming that disruption of immune surveillance may facilitate the emergence of metastatic clones [6].

Moreover, in patients with HCV-related cirrhosis, Conti et al. suggested that DAA-induced resolution of $\mathrm{HCV}$ infection does not seem to reduce occurrence of HCC and patients previously treated for HCC still have a high risk of tumor recurrence, in the short term [7].

However, it is important to consider that a recent meta-analysis showed that pooled estimates of the 2-year recurrence and 5-year survival rates in $\mathrm{HCV}$ cirrhotic patients with Barcelona Clinic for Liver Cancer HCC cured with resection or ablation were both about 50\% [8], while another recent study indirectly suggests that DAA could improve overall survival of HCC through long-term preservation of liver function, resulting in a lower cirrhosis-related mortality and a greater change of receiving curative treatments [9].

In this line, Calvaruso et al. observed that the early occurrence of HCC in cirrhotic patients treated with DAAs remained similar to that reported in historical cohorts of untreated patients and that the risk of $\mathrm{HCC}$ was higher in patients with Child-Pugh B cirrhosis and in patients without sustained virological response [10].

Cirrhotic patients remain the main target of HCC early diagnosis by performing semiannual surveillance 1 by abdominal ultrasound, even if a contrast-enhanced polyphasic CT scan or hepatospecific contrast-enhanced MRI is mandatory as a diagnostic confirmation; planned biopsies of focal lesions are needed for doubtful cases.

HCC staging systems have also been under discussion. Over the last 20 years, a variety of prognostic systems have been proposed in an attempt to address the interrelationship of prognostic factors among HCC patients and the complexity of HCC treatment, but no single system for HCC has been universally adopted.

Based on these considerations, Vitale et al. (Padova, Italy) described a new prognostic system developed and validated by Farinati and colleagues for HCC patients including both a tumor staging and a prognostic score: the Italian Liver Cancer dataset [11]. This prognostic system includes both a tumor staging score, which stratifies patients with HCC into six main stages (0, A, B1, B2, B3 and C), and a prognostic score that integrates Italian Liver Cancer tumor staging, Eastern Cooperative Oncology Group performance status, Child-Pugh score and $\alpha$-fetoprotein. It showed a strong ability to predict individual survival in European and Asian populations [12] and introduced the concept of 'therapeutic hierarchy' suggesting the relevance of the multidisciplinary approach in the management of HCC.

\section{Early disease therapeutic management}

In the field of curative therapies for HCC, thermal ablation remains a widely used option especially for the treatment of smaller nodules $(<3 \mathrm{~cm})$. In recent years, microwave ablation has partially replaced radiofrequency ablation in many centers given the greater ease of use and its excellent results [13]. In nodules less than $3 \mathrm{~cm}$, surgical resection and ablative treatments are similar in terms of results obtained. For larger individual nodules $(3-5 \mathrm{~cm})$, surgical resection seems to favor a longer life expectancy and better cost-effectiveness than ablation; otherwise, in patients with $2-3$ nodules less than $3 \mathrm{~cm}$, life expectancy is similar for the two treatments although ablation showed a better cost-effectiveness [14].

Moreover, it has been discussed if the sequential use and/or the combination of different therapeutic methods, such as thermal ablation and TACE, may favor better results in terms of response rate and survival in selected patients. Also debated was the role of liver transplantation in the context of healing therapies because the Milan criteria are often considered very restrictive, so that more expansive criteria have been proposed albeit requiring further validation.

In this setting, locoregional therapies are often also used to prevent disease progression or to achieve a downstaging while the patient is shortlisted for transplantation.

Among intra-arterial treatments, TACE is still the gold standard for patients with intermediate stage disease, HCC [15]. Nevertheless, the BCLC $\mathrm{B}$ stage comprises a very heterogeneous population of patients with many differences in tumor characteristics and liver function. In many cases, a second course of TACE is needed to obtain complete response; according to the position paper of the Italian Association for the Study of the Liver (Associazione Italiana per lo Studio del Fegato, [AISF]), therapy with sorafenib may be considered in case of failure of TACE (i.e., lack of objective response of the treated lesions after two procedures) [16]. 
A careful selection of patients amenable to TACE has to be performed, taking into account tumor stage, liver function, patient's comorbidity and performance status.

To this end, Peck-Radosavljevic (Vienna) enounced different selection score systems including Assessment for Retreatment with TACE (ART) that evaluates whether HCC patients can benefit from TACE retreatments: TACE has a good prognostic effect on patients with ART score of $0-1.5$, while patients with an ART score $\geq 2.5$ might experience minor or even no survival benefits [17].

In recent years, transarterial radio-embolization (TARE) with yttrium-90 achieved a promising role in terms of efficacy and tolerability in HCC treatment as explained by Bhoori (Milan): this method is currently indicated in locally advanced HCC or in large nodule HCC even if a few studies evaluated its role also in early stages [18].

The benefits of combined systemic and liverdirected treatments in inoperable intermediateor advanced-stage HCC need to be defined. A randomized controlled trial compared TARE with yttrium-90 resin followed by sorafenib with sorafenib alone (SORAMIC study) in patients who were poor candidates for transarterial (chemo) embolization with preserved liver function (Child-Pugh $\leq$ B7) and Eastern Cooperative Oncology Group performance status $<2$, showing no difference in toxicities. Phase III studies results are needed to confirm the efficacy and safety of the combination of TARE and sorafenib in HCC treatment [19].

\section{Systemic therapy}

This topic was presented extensively at the congress. Sorafenib represents the 'gold standard' reference for clinical trials designed to evaluate the efficacy of new systemic therapies [20]: some Phase III studies have compared the efficacy of new molecules as sunitinib, brivanib, linifanib, erlotinib, versus sorafenib [21]. Nevertheless, none of them have been superior to sorafenib.

In the second-line setting, brivanib, ramucirumab and everolimus have been studied with no evidence of effectiveness. On the contrary a recently published Phase III randomized double-blind study, the RESORCE trial (NCT01774344), assessed regorafenib as the only systemic treatment shown to provide survival benefit in HCC patients progressing on sorafenib treatment compared with best supportive care, with a median overall survival of 10.6 months for regorafenib versus 7.8 months for placebo [22]. At present, regorafenib is the only systemic treatment shown to provide survival benefit in HCC patients progressing on sorafenib treatment. Future trials should explore different agents in second-line setting in patients who do not tolerate sorafenib, and in third-line setting for patients who fail or who do not tolerate the sequence of sorafenib and regorafenib.

While waiting for these trials and regorafenib approval in all countries, there is consensus on continuing sorafenib in patients with radiological progressive but clinically stable disease also because sorafenib interruption could result in a rapid and symptomatic disease progression [23].

Further developments in HCC therapies were also discussed. Immunotherapy in particular represents a promising treatment approach: nivolumab, anti-PD-1 monoclonal antibody (programmed death-1), appears the most promising immunotherapy [24].

Currently a Phase III study evaluating nivolumab versus sorafenib as first-line treatment in patients with advanced HCC is ongoing [24].

Last, HCC remains a complex and heterogeneous disease with wide prognosis and HCC patients should be referred to centers with collaborative, coordinate and multidisciplinary teams that are best suited to offer the best care, to facilitate and to speed patient management.

\section{Financial \& competing interests disclosure}

The authors have no relevant affliations or financial involvement with any organization or entity with a financial interest in or financial conflict with the subject matter or materials discussed in the manuscript. This includes employment, consultancies, honoraria, stock ownership or options, expert testimony, grants or patents received or pending, or royalties.

No writing assistance was utilized in the production of this manuscript.

\section{References}

1 deOliveria Andrade LJ, D’Oliveira A, Carvalho Melo R, De Souza EC, Costa Silva
CA, Paraná R. Association between hepatitis $\mathrm{C}$ and hepatocellular carcinoma. J. Glob. Infect. Dis. 1, 33-37 (2009).
2 Parkin DM, Bray F, Ferlay J, Pisani P. Global cancer statistics, 2002. CA Cancer J Clin. 55, 74-108 (2005). 
3 Llovet JM, Burroughs A, Bruix J. Hepatocellular carcinoma. Lancet 362, 1907-1917 (2003).

4 Bruix J, Sherman M. Management of hepatocellular carcinoma: an update. Hepatology 53(3), 1020-1022 (2011).

5 Triolo M, Della Corte C, Colombo M et al. Impact of HBV therapy on the incidence of hepatocellular carcinoma. Liver Int. 34(Suppl. 1), 139-145 (2014).

6 Reig M, Mariño Z, Perelló C et al. Unexpected high rate of early tumor recurrence in patients with $\mathrm{HCV}$-related HCC undergoing interferon-free therapy. J. Hepatol. 65(4), 719-726 (2016).

7 Conti F, Buonfiglioli F, Scuteri A et al. Early occurrence and recurrence of hepatocellular carcinoma in HCV-related cirrhosis treated with direct-acting antivirals. J. Hepatol. 65(4), 727-733 (2016).

8 Cabibbo G, Petta S, Barbàra M et al. A meta-analysis of single HCV-untreated arm of studies evaluating outcomes after curative treatments of HCV-related hepatocellular carcinoma. Liver Int. doi:10.1111/liv.13357 (2017) (Epub ahead of print).

9 Cabibbo G, Petta S, Barbara M et al. Hepatic decompensation is the major driver of death in HCV-infected cirrhotic patients with successfully treated early hepatocellular carcinoma. J. Hepatol. doi:10.1016/j. jhep.2017.01.033 (2017) (Epub ahead of print).

10 Calvaruso V, Cabibbo G, Cacciola I et al. Early occurrence of hepatocellular carcinoma (HCC) in patients with HCV cirrhosis treated with direct-acting antivirals (DAAs). Dig. Liver Dis. 49(1), e60 (2017).

11 Vitale A, Farinati F, Huo TL et al. The concept of therapeutic hierarchy for patients with hepatocellular carcinoma: a multicenter cohort study. Dig. Liver Dis. 49(1), e4-e5 (2017).

12 Farinati F, Vitale A, Spolverato G et al. Development and validation of a new prognostic system for patients with hepatocellular carcinoma. PLoS Med. 13(4), e1002006 (2016).

13 Sacco R, Antonucci M, Bresci G et al. Curative therapies for hepatocellular carcinoma: an update and perspectives. Expert Rev. Anticancer Ther. 16(2), 169-175 (2016).

14 Cucchetti A, Piscaglia F, Cescon M et al. Cost-effectiveness of hepatic resection versus percutaneous radiofrequency ablation for early hepatocellular carcinoma. J. Hepatol. 59(2), 300-307 (2013).

15 Sacco R, Antonucci M, Bargellini I, Marceglia S, Mismas V, Cabibbo G. Transarterial chemoembolization and sorafenib in patients with intermediate-stage hepatocellular carcinoma: time to enter routine clinical practice? Future Oncol. 11(17), 2371-2373 (2015).

16 Italian multisocietarie recommendations for clinical management of patients with hepatocarcinoma. www.aiom.it/professionisti

17 Yin W, Ye Q, Wang F et al. FART score and hepatocellular carcinoma: an appraisal of its applicability. Clin. Res. Hepatol. Gastroenterol. 40 (6), 705-714 (2016).
18 Sacco R, Conte C, Tumino E et al. Transarterial radioembolization for hepatocellular carcinoma: a review. J. Hepatocell Carcinoma 3, 25-29 (2016).

19 Ricke J1, Bulla K, Kolligs F et al. Safety and toxicity of radioembolization plus Sorafenib in advanced hepatocellular carcinoma: analysis of the European multicentre trial SORAMIC. Liver Int. 35(2), 620-626 (2015).

20 Cabibbo G1, Petta S, Maida M, Cammà C. Sorafenib for hepatocellular carcinoma: from randomized controlled trials to clinical practice. Dig. Dis. 33(5), 668-674 (2015).

21 Llovet JM1, Hernandez-Gea V. Hepatocellular carcinoma: reasons for Phase III failure and novel perspectives on trial design. Clin. Cancer Res. 20(8), 2072-2079 (2014).

22 Bruix J, Qin S, Merle P et al. Regorafenib for patients with hepatocellular carcinoma who progressed on sorafenib treatment (RESORCE), a randomised, double-blind, placebo-controlled, Phase III trial. Lancet 389(10064), 56-66 (2017).

23 Iavarone M, Cabibbo G, Biolato $\mathrm{M}$ et al. Predictors of survival in patients with advanced hepatocellular carcinoma who permanently discontinued sorafenib. Hepatology 62 (3), 784-791 (2015).

24 Kudo M. Immune checkpoint inhibition in hepatocellular carcinoma: basics and ongoing Clin. Trials Oncol. 92(Suppl. 1), 50-62 (2017). 